CLINICAL STUDY

\title{
Pioglitazone increases bone marrow fat in type 2 diabetes: results from a randomized controlled trial
}

\author{
Andrew Grey ${ }^{1}$, Vaughan Beckley ${ }^{2, *}$, Anthony Doyle ${ }^{2, *}$, Sheryl Fenwick ${ }^{1}$, Anne Horne ${ }^{1}$, Greg Gamble ${ }^{1}$ and \\ Mark Bolland ${ }^{1}$ \\ Departments of ${ }^{1}$ Medicine and ${ }^{2}$ Anatomy with Radiology, University of Auckland, Private Bag 92019, Auckland 1142, New Zealand \\ (Correspondence should be addressed to A Grey; Email: a.grey@auckland.ac.nz) \\ *(V Beckley and A Doyle contributed equally to this work)
}

\begin{abstract}
Objective: To determine the effect of pioglitazone on bone marrow fat in humans.

Design: Twenty participants in a double-blind, randomized, placebo-controlled trial of the skeletal effects of pioglitazone $30 \mathrm{mg}$ daily in type 2 diabetes mellitus (T2DM) entered a 6-month substudy evaluating bone marrow fat. Main outcome measures were bone marrow fat in lumbar spine (L4) and proximal femur (intertrochanteric region), measured using magnetic resonance (MR) imaging, and bone mineral density (BMD), measured using dual-energy X-ray absorptiometry.

Results: After 6 months, change in the fourth lumbar vertebra (L4) bone marrow lipid fraction, assessed using two different methods, was greater in the pioglitazone group than in the placebo group (Dixon method: mean $(95 \% \mathrm{CI})$ change from baseline pioglitazone $1.3 \%(-0.3,2.9)$, placebo $-0.2 \%$ $(-0.8,0.4), P=0.06$; MR spectroscopy: pioglitazone $2.5 \%(0.4,4.7)$, placebo $-1.1 \%(-3.7,1.4)$, $P=0.02)$. Similarly, the change in lipid fraction in the intertrochanteric region was greater in the pioglitazone group (Dixon method: mean $(95 \% \mathrm{CI})$ change from baseline pioglitazone $1.3 \%(0.6,1.9)$, placebo $-0.8 \%(-1.8,0.2), P=0.001)$. Within the pioglitazone group, there was no evidence of a significant relationship between change in marrow lipid fraction and BMD.

Conclusions: Short-term treatment with pioglitazone increases bone marrow fat in patients with T2DM.
\end{abstract}

European Journal of Endocrinology 166 1087-1091

\section{Introduction}

Thiazolidinediones (TZDs) are agonists of the nuclear transcription factor peroxisome proliferator-activated receptor-gamma (PPAR $\gamma$ (PPARG)) that increase insulin sensitivity and improve glycemic control in type 2 diabetes mellitus (T2DM) (1). PPAR $\gamma$ is also a molecular switch that regulates mesenchymal stem cell fate, favoring adipogenesis over osteoblastogenesis (2). Several preclinical studies reported increased bone marrow fat in parallel with decreased bone mineral density (BMD) after treatment with TZDs (3). In humans, TZDs decrease BMD and increase fracture risk $(4,5)$. The mechanism by which this increased skeletal fragility is mediated is unclear, with evidence for both decreased bone formation (6) and increased bone resorption (7).

Evaluating the effect of TZDs on marrow fat in humans is important for two reasons. First, it is plausible that increased bone marrow fat induced by TZDs might detrimentally affect BMD. Evidence from in vitro studies suggests that adipocytes adversely influence bone cell function in a paracrine manner (8), prospective studies on animals report a contemporaneous increase in marrow fat and decrease in BMD (9), and correlative studies in humans suggest an inverse relationship between bone marrow fat and BMD (10). Second, it has been suggested that changes in bone marrow fat might artifactually alter measurement of BMD by dualenergy X-ray absorptiometry (DXA) (11).

Currently, however, data on the effect of TZDs on bone marrow fat in humans are limited. Harslof et al. (12) reported that 6 months of treatment with rosiglitazone decreased both BMD and bone marrow fat in healthy postmenopausal women, the latter finding being surprising given the consistent evidence of increased marrow fat in TZD-treated animals. To further study the effect of TZDs on bone marrow fat, we assessed marrow adiposity in the proximal femur and lumbar spine over 6 months in a subset of participants in a randomized controlled trial of the skeletal effects of pioglitazone in T2DM.

\section{Materials and methods}

\section{Participants and protocol}

The first 20 consenting participants enrolling in a randomized placebo-controlled trial of pioglitazone, 
$30 \mathrm{mg}$ daily, entered a substudy to assess the effect of the intervention on bone marrow fat. The primary endpoint of the parent trial was change in lumbar spine BMD at 12 months. Participants were men and women $>30$ years with T2DM. Exclusion criteria included congestive heart failure New York Heart Association grade $\geq 2$, BMD $T$ score at hip or spine $<-2$, previous fracture of hip, vertebra, forearm or humerus, chronic liver or renal disease, diseases or medications known to affect bone metabolism, and body weight $>120 \mathrm{~kg}$. Randomization to daily treatment with either pioglitazone $30 \mathrm{mg}$ or a matching placebo was undertaken using a variable block size schedule based on computergenerated random numbers, stratified for age, menopausal status, and gender. Only the statistician had access to treatment allocation, and he had no contact with participants. All the other study personnel and participants were blinded to treatment allocation throughout. The study received ethical approval from the Northern Y Regional Ethics Committee and was registered with the Australian New Zealand Clinical Trials Registry, ACTRN12607000610437, date of registration 28/11/07. All participants gave written informed consent.

\section{Bone marrow fat}

Lumbar spine and bilateral proximal femoral magnetic resonance (MR) imaging (MRI) was performed using a 1.5 Tesla MR scanner (Siemens Avanto, Erlangen, Germany). Marrow fat was evaluated by two distinct techniques.

Spectroscopy The L4 vertebral body was identified using sagittal and coronal plane localizer T1 turbo spin echo (TSE) images. A single $15 \mathrm{~mm}^{3}$ voxel was positioned within the mid-anterocentral vertebral marrow space to avoid inclusion of cortical bone or the endplates. ${ }^{1} \mathrm{H}-\mathrm{MR}$ spectroscopy was performed using a modified (no water suppression) stimulated echo acquisition mode single voxel sequence, with the following parameters: repetition time (TR) $5000 \mathrm{~ms}$, echo time (TE) $20 \mathrm{~ms}, 1024$ data points, bandwidth $1000 \mathrm{~Hz}$, and acquisition time $5 \mathrm{~min} 40 \mathrm{~s}$ (13). Free induction decays were analyzed using the Hankel Lanczos Squares Singular Value Decomposition algorithm, based on the Lanczos algorithm for the estimation of spectral components in the time domain (jMRUI software v4.0, Graveron-Demilly et al., Claude Bernard University, Lyon, France). A two-component analysis was performed to represent the primary water and lipid peaks, with 2048 point and 1024 row Hankel matrix parameters. The integrals of the peaks for water $(\mathrm{Pw})$ and fat $(\mathrm{Pf})$ were recorded. The lipid fraction was calculated as $\mathrm{Pf} /(\mathrm{Pf}+\mathrm{Pw})$. The lipid fraction provides a value between 0 (no fat) and 1 (only fat, no water).

Quantitative chemical shift A modified Dixon technique (14) was used to measure the fat fraction at the L4 vertebral body and the intertrochanteric regions.
In-phase and opposed-phase $\mathrm{T} 1$ gradient echo 3D sequences were performed with the following parameters: TR $7.03 \mathrm{~ms}$, TE $2.38 \mathrm{~ms}, 4.76 \mathrm{~ms}$, 3NSA, $1.0 \times 1.0 \times 4.0 \mathrm{~mm}$ voxel size, and contiguous axial stacks to cover the whole region of interest (ROI). Water and fat images were generated from the in- and outof-phase images using the Siemens (B17 version) software. T1 TSE axial images were acquired to cover the ROI matched to the Dixon imaging, using parameters TR $550 \mathrm{~ms}$ and TE $10 \mathrm{~ms}$. Axial multislice imaging was also performed through the hips, including both hip joints and the proximal femora extending to a few centimeters below the lesser trochanters. At the L4 vertebral body, five slices were selected to represent the most central marrow space of the body, chosen to avoid the vertebral endplate or intervertebral disc. At the proximal femora, ten slices were selected, starting at the level of the lesser trochanter and proceeding proximally. A brush ROI was painted at each slice within the margins of the low signal cortex on the fat-only image; this ROI was then eroded by four pixels in the L4 images and three pixels in the femoral neck images in order to exclude a uniform amount of the peripheral marrowcortical interface. The ROI was directly copied into the water-only image to ensure that the same area in each corresponding slice was sampled. The pixel values were averaged across the total number of slices sampled in both the fat-only (Mf) and water-only (Mw) series. The lipid fraction was calculated as $\mathrm{Mf} /(\mathrm{Mf}+\mathrm{Mw})$.

\section{Bone mineral density}

BMD was measured at baseline and 6 months at the lumbar spine (L1-L4) and dual proximal femur using a Lunar Prodigy DXA (GE Lunar, Madison, WI, USA). The coefficients of variation for measurement of total hip and lumbar spine BMDs in our laboratory are 1.1 and $1.4 \%$ respectively.

\section{Statistical analysis}

Data were analyzed using procedures of SAS (v9.2; SAS Institute, Inc., Cary, NC, USA). Changes from baseline in bone marrow fat at each site, and BMD at each site, were compared between study groups using Student's $t$-test for unpaired data. Correlations between change in bone marrow fat and change in BMD were performed using Spearman's correlation coefficient.

\section{Results}

The baseline characteristics of the participants in the substudy cohort are shown in Table 1. Mean compliance with study medication, assessed by tablet count, was $89 \%$ in the pioglitazone group and $96 \%$ in the placebo group. During the study, $\mathrm{HbA1c}$ declined in the 
Table 1 Baseline characteristics of the study participants. Data are mean (S.D.) or $n$.

\begin{tabular}{lcc}
\hline Variable & $\begin{array}{c}\text { Placebo } \\
(n=10)\end{array}$ & $\begin{array}{c}\text { Pioglitazone } \\
(n=10)\end{array}$ \\
\hline Age (years) & $57.9(15.2)$ & $61.9(10.0)$ \\
Gender $(\mathrm{M} / \mathrm{F})$ & $5 / 5$ & $6 / 4$ \\
Weight $(\mathrm{kg})$ & $91.7(12.8)$ & $90.1(14.9)$ \\
BMl $\left(\mathrm{kg} / \mathrm{m}^{2}\right)$ & $33.2(4.1)$ & $31.2(4.7)$ \\
HbA1c $(\%)$ & $7.1(1.0)$ & $7.6(2.1)$ \\
Diabetes medications & & \\
Metformin & 9 & 5 \\
Sulfonylurea & 3 & 1 \\
Insulin & 1 & $1.27(0.18)$ \\
BMD L4 (g/cm $\left.{ }^{2}\right)$ & $1.46(0.30)$ & $0.91(0.14)$ \\
BMD trochanter $\left(\mathrm{g} / \mathrm{cm}^{2}\right)$ & $0.95(0.14)$ & $73(8)$ \\
LF L4 $(\%)$ & $72(6)$ & $90(6)$ \\
LF intertrochanteric ${ }^{\mathrm{a}}(\%)$ & $89(6)$ & \\
\hline
\end{tabular}

LF, lipid fraction. ${ }^{\star} P<0.05$ vs placebo.

aDixon method.

pioglitazone group (mean $(95 \%$ CI) change from baseline $-0.28 \%(-0.02,-0.54), P=0.03)$ and was unchanged in the placebo group (mean $(95 \% \mathrm{CI})$ change from baseline $-0.04 \%(-0.45,0.37), P=0.83)$.

Figure 1 shows the results of the bone marrow fat analyses. At L4, the change in lipid fraction was greater in the pioglitazone group than in the placebo group, irrespective of the measurement technique applied (Dixon method: mean $(95 \% \mathrm{CI})$ change from baseline pioglitazone $1.3 \%(-0.3,2.9)$, placebo $-0.2 \%(-0.8$, 0.4), $P=0.06$; magnetic resonance spectroscopy method: pioglitazone $2.5 \%(0.4,4.7)$, placebo $-1.1 \%$ $(-3.7,1.4), P=0.02)$ (Fig. $1 \mathrm{~A}$ and C). Similarly, the change in lipid fraction in the left intertrochanteric region was greater in the pioglitazone group (mean (95\% CI) change from baseline pioglitazone $1.3 \%(0.6$, $1.9)$, placebo $-0.8 \%(-1.8,0.2), P=0.001)$ (Fig. 1B). Similar findings were present at the right intertrochanteric site (data not shown). Within the pioglitazone group, there was no evidence that the change in marrow fat differed by gender. Mean (95\% CI) change in L4 lipid fraction was $1.6 \%(-0.4,3.5)$ in men and $0.9 \%$ $(-3.8,5.6)$ in women; change in left intertrochanteric lipid fraction was $1.4 \%(0.6,2.2)$ in men and $1.1 \%$ $(-0.6,2.7)$ in women $(P>0.5$ at each site $)$.

It has been suggested that change in bone marrow adiposity might artifactually influence BMD measurements performed using DXA (11). We used data from the pioglitazone group to examine the relationship between change in marrow fat and BMD. The relationship between these variables was not significant at either site $(\mathrm{L} 4, r=-0.18, P=0.63$; trochanter, $r=-0.02, P=0.97)$.

Some evidence also suggests that HbA1c levels are positively related to marrow fat in patients with T2DM (15). We did not find a significant relationship between baseline $\mathrm{HbA} 1 \mathrm{c}$ levels and marrow fat at either site (L4, $r=-0.24, P=0.31$; trochanter, $r=-0.17$,
$P=0.48)$. Nor was there evidence for a relationship between change in $\mathrm{HbAlc}$ and marrow fat in the pioglitazone group (L4, $r=0.25, P=0.49$; trochanter, $r=0.35, P=0.33)$.

\section{Discussion}

This study provides evidence that treatment with pioglitazone increases bone marrow fat in people with T2DM. We assessed marrow fat at two skeletal sites,
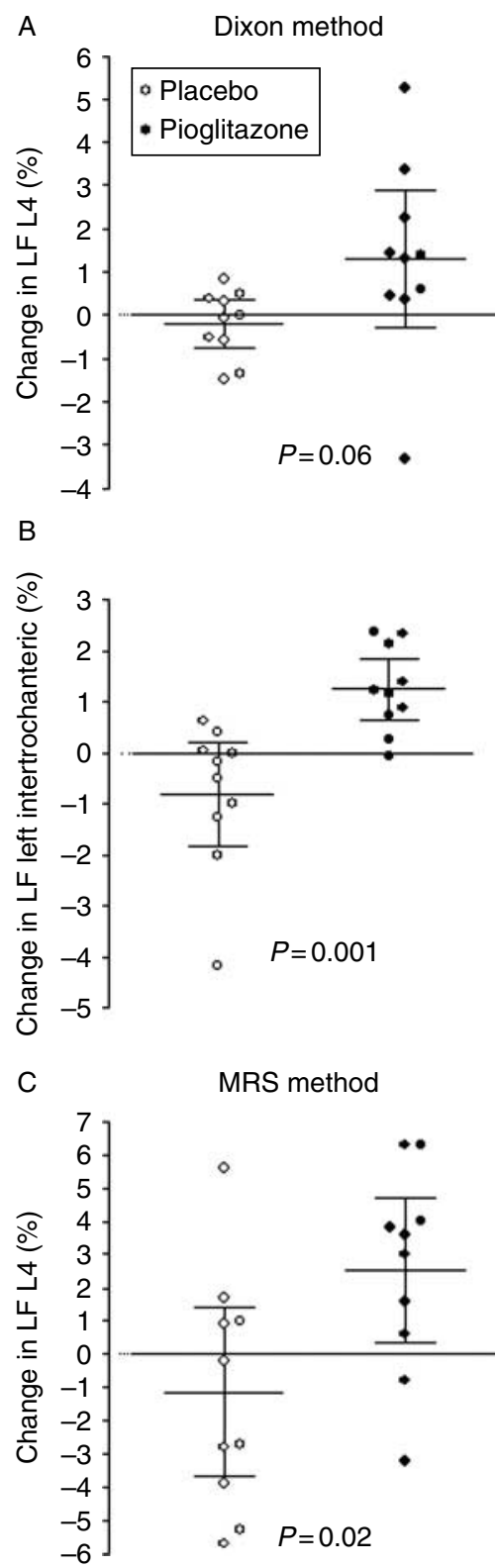

Figure 1 Effect of pioglitazone on bone marrow fat in L4 and left intertrochanteric region. Each symbol represents the change from baseline value in a study participant. Horizontal bars are mean and $95 \%$ CI. LF, lipid fraction. 
the proximal femur and the lumbar spine, using two distinct MRI-based techniques and found significant increases in the marrow adiposity in each instance. The results are consistent with in vitro and preclinical studies that suggest that activation of PPAR $\gamma$ signaling preferentially directs mesenchymal stem cells into the adipocyte lineage at the expense of osteoblastogenesis (4), but to our knowledge are the first data that demonstrate a TZD-induced increase in marrow adiposity in humans. Our findings are different to those of Harslof et al. (12), who reported that 6 months of rosiglitazone treatment decreased bone marrow fat in the lumbar spine of healthy postmenopausal women, assessed using MRI proton spectroscopy. The reason(s) for the divergent results of the two studies is not clear. It is possible that pioglitazone and rosiglitazone differ in their effects on marrow adipogenesis in vivo. The preclinical literature does not include any studies on animals treated with pioglitazone in which marrow fat was evaluated (3). It is also possible that diabetic subjects respond differently to TZDs than nondiabetic subjects. Our study included men, while that of Harslof et al. included only women. The skeletal fragility induced by TZDs is more readily apparent in women $(5,16)$. We found no consistent evidence that gender modulated the effect of pioglitazone on marrow fat, but the number of participants of each gender was small.

The current findings may have relevance to two important issues. First, it is possible that increased marrow adiposity contributes to the increased skeletal fragility observed in clinical studies of TZDs. Co-culture experiments demonstrate that adipocytes induce osteoblast apoptosis via a paracrine mechanism (8) and inverse relationships between marrow fat and BMD have been reported in prospective studies on animals (9) and cross-sectional studies on humans (10). Decreased bone formation has been reported in both preclinical and clinical studies on TZDs (3). Second, ex vivo experiments suggest that increased marrow fat might artifactually decrease BMD measured by DXA (11). Whether this applies in vivo is uncertain - genetic ablation of marrow fat in mice does not alter BMD measured by DXA (17), and the current results, failing to show a consistent inverse relationship between changes in marrow fat and BMD during TZD therapy, suggest that the findings of decreased BMD in TZD-treated subjects are not artifactual. The latter notion is supported by recent evidence that rosiglitazone decreases BMD measured using quantitative computed tomography (18) and by in vivo modeling studies that suggest that changes in marrow fat of the magnitude we observed are unlikely to alter BMD measured by DXA (19).

Our study has limitations. The number of participants was small, and evaluation of bone marrow fat was not the primary endpoint of the study. We only studied participants after 6 months of exposure to pioglitazone. Direct confirmation of the changes in bone marrow fat identified radiologically was not undertaken. Neither was it possible to assess the metabolic activity or composition of marrow fat after TZD exposure.

In summary, the current study provides preliminary evidence that bone marrow fat increases during shortterm treatment with pioglitazone, suggesting a potential means by which TZDs might decrease osteoblast function and thereby BMD. The absence of an inverse relationship between change in marrow fat and that in BMD suggests that DXA measurements in patients treated with TZDs provide a valid estimate of BMD.

\section{Declaration of interest}

The authors declare that there is no conflict of interest that could be perceived as prejudicing the impartiality of the research reported.

\section{Funding}

Funding support was from the Health Research Council of New Zealand. Trial registration: actr.org.au Identifier: ACTRN 12607000610437 , date of registration 28/11/07.

\section{References}

1 Yki-Jarvinen H. Thiazolidinediones. New England Journal of Medicine 2004351 1106-1118. (doi:10.1056/NEJMra041001)

2 Pei L \& Tontonoz P. Fat's loss is bone's gain. Journal of Clinical Investigation 2004113 805-806. (doi:10.1172/JCI21311)

3 Grey A. Skeletal consequences of thiazolidinedione therapy. Osteoporosis International $2008 \quad 19$ 129-137. (doi:10.1007/ s00198-007-0477-y)

4 Grey AB. Thiazolidinedione-induced skeletal fragility mechanisms and implications. Diabetes, Obesity and Metabolism 200911 275-284. (doi:10.1111/j.1463-1326.2008.00931.x)

5 Douglas IJ, Evans SJ, Pocock S \& Smeeth L. The risk of fractures associated with thiazolidinediones: a self-controlled case-series study. PLoS Medicine 20096 e1000154. (doi:10.1371/journal. pmed.1000154)

6 Grey A, Bolland M, Gamble G, Wattie D, Horne A, Davidson J \& Reid IR. The peroxisome proliferator-activated receptor-gamma agonist rosiglitazone decreases bone formation and bone mineral density in healthy postmenopausal women: a randomized, controlled trial. Journal of Clinical Endocrinology and Metabolism 200792 1305-1310. (doi:10.1210/jc.2006-2646)

7 Gruntmanis U, Fordan S, Ghayee HK, Abdullah SM, See R, Ayers CR \& McGuire DK. The peroxisome proliferator-activated receptor-gamma agonist rosiglitazone increases bone resorption in women with type 2 diabetes: a randomized, controlled trial. Calcified Tissue International $2010 \mathbf{8 6} 343-349$. (doi:10.1007/ s00223-010-9352-5)

8 Elbaz A, Wu X, Rivas D, Gimble JM \& Duque G. Inhibition of fatty acid biosynthesis prevents adipocyte lipotoxicity on human osteoblasts in vitro. Journal of Cellular and Molecular Medicine 201014 982-991. (doi:10.1111/j.1582-4934.2009.00751.x)

9 Devlin MJ, Cloutier AM, Thomas NA, Panus DA, Lotinun S, Pinz I, Baron R, Rosen CJ \& Bouxsein ML. Caloric restriction leads to high marrow adiposity and low bone mass in growing mice. Journal of Bone and Mineral Research 201025 2078-2088. (doi:10.1002/jbmr.82)

10 Rosen CJ \& Bouxsein ML. Mechanisms of disease: is osteoporosis the obesity of bone? Nature Clinical Practice Rheumatology 20062 35-43. (doi:10.1038/ncprheum0070)

11 Chappard D, Moquereau M, Mercier P, Gallois Y, Legrand E, Basle MF \& Audran M. Ex vivo bone mineral density of the wrist: influence of medullar fat. Bone 200434 1023-1028. (doi:10.1016/j.bone.2004.02.002) 
12 Harslof T, Wamberg L, Moller L, Stodkilde-Jorgensen H, Ringgaard S, Pedersen SB \& Langdahl BL. Rosiglitazone decreases bone mass and bone marrow fat. Journal of Clinical Endocrinology and Metabolism 201196 1541-1548. (doi:10.1210/jc.2010-2077)

13 Schellinger D, Chin S, Fertikh D, Lee J, Lauerman W, Henderson F \& Davis B. Normal lumbar vertebrae: anatomic, age and sex variance in subjects at proton MR spectroscopy initial experience. Radiology 2000215 910-916.

14 Dixon WT. Simple proton spectroscopic imaging. Radiology 1984 153 189-194.

15 Baum T, Yap SP, Karampinos DC, Nardo L, Kuo D, Burghardt AJ, Masharani UB, Schwartz AV, Li X \& Link TM. Does vertebral bone marrow fat content correlate with abdominal adipose tissue, lumbar spine bone mineral density, and blood biomarkers in women with type 2 diabetes mellitus? Journal of Magnetic Resonance Imaging 201235 117-124. (doi:10.1002/jmri.22757)

16 Loke YK, Singh S \& Furberg CD. Long-term use of thiazolidinediones and fractures in type 2 diabetes: a meta-analysis. CMAJ: Canadian Medical Association Journal $200918032-39$. (doi:10.1503/cmaj.080486)
17 Justesen J, Mosekilde L, Holmes M, Stenderup K, Gasser J, Mullins JJ, Seckl JR \& Kassem M. Mice deficient in 11ßhydroxysteroid dehydrogenase type 1 lack bone marrow adipocytes, but maintain normal bone formation. Endocrinology 2004 145 1916-1925. (doi:10.1210/en.2003-1427)

18 Bilezikian JP, Eastell R, Nino AJ, Northcutt AR, Kravitz B, Paul G, Cobitz AR, Josse R \& Fitzpatrick L. Rosiglitazone reduced BMD in women with type 2 diabetes and the effect was attenuated after switching to metformin. Journal of Bone and Mineral Research 2011 26 (Suppl 1).

19 Blake GM, Griffith JF, Yeung DKW, Leung PC \& Fogelman I. Effect of increasing vertebral marrow fat content on BMD measurement, $T$ score status and fracture risk prediction by DXA. Bone $2009 \mathbf{4 4}$ 495-501. (doi:10.1016/j.bone.2008.11.003)

Received 16 December 2011

Revised version received 22 February 2012

Accepted 9 March 2012 